\title{
Cultures et élevage : entre échelle et gamme de production, quels enjeux économiques et éthiques?
}

Oléagineux, Corps Gras, Lipides. Volume 11, Numéro 4, 256-60, JUILLET-OCTOBRE 2004, AGRICULTURE / ÉLEVAGE : NORD-SUD

Auteur(s) : Dominique VERMERSCH

Département Economie rurale et gestion, Pôle agronomique, 65, rue de Saint-Brieux, C5 84 215, F 35042 Rennes Cedex

\section{ARTICLE}

Y a-t-il encore un avenir aux politiques agricoles ? La question mérite d'être posée dans un contexte de libéralisation accrue des échanges commerciaux, et où le mot-clé mythique de découplage fait quasiment figure de mot de la fin, voire de « fin de l'Histoire » de ces politiques. Cela dit, il semble bien que ce prêche libéral tourne aujourd'hui à une sorte de ritournelle vieillissante, qui se raidit à mesure de son caractère idéologique plus prononcé. Plus personne ou presque ne croit raisonnablement que les trop fameux "gains de bien-être " issus de la libéralisation des marchés agricoles soient la réponse à tout et puissent ne serait-ce que compenser les " pertes de bien-être " associées au démantèlement des agricultures locales et des diverses aménités coproduites. D'un autre côté, il ne s'agit pas non plus de se leurrer : le discours du « local », du " small is beautiful » ou encore des nostalgies champêtres n'est pas non plus exempt d'idéologie.Entre progressisme et conservatisme, les deux approches précédentes se confrontent depuis quelques années autour du concept de multifonctionnalité. Rapportée à l'agriculture, elle stipule l'existence d'une gamme de produits très divers, élaborés conjointement, certains d'entre eux étant des externalités et/ou des biens publics ${ }^{\underline{1}}$ qui ne peuvent donc être produits et alloués efficacement par le marché. La multifonctionnalité agricole se définit également d'un point de vue normatif, au vu des fonctions multiples assignées à l'agriculture qui deviennent alors autant d'objectifs d'action des pouvoirs publics. Au-delà de sa fonction première de production de nourriture et de fibres, l'activité agricole peut aussi créer des paysages, procurer des bénéfices environnementaux comme l'entretien des sols, la gestion durable des ressources naturelles renouvelables et la protection de la biodiversité; et contribuer à la viabilité socio-économique des zones rurales [1].En réalité, toutes ces fonctions ne sont pas nouvelles mais bénéficient aujourd'hui d'un regain d'intérêt du fait justement que les modes contemporains de production agricole les ont quelque peu délaissées, à la faveur notamment d'une hétéronomie croissante des modes de production agricole. Cette hétéronomie est à l'origine d'une dissociation des productions, comme celle que nous observons entre cultures et élevage, dissociation à l'origine de la plupart des nuisances agri-environnementales et d'une préférence pour l'échelle plutôt que pour la gamme de production. C'est ce que nous analyserons dans un premier temps, ce qui nous permettra ensuite de mieux comprendre comment ce mouvement de découplage des productions a précipité voire facilité une politique de découplage des aides publiques à l'agriculture dont nous n'avons peut-être pas anticipé aujourd'hui tous les travers. C'est à partir de ce constat que l'article débouche sur une réflexion éthique, indispensable nous semble-t-il à une réorientation pertinente des politiques agricoles [2-4]. 


\section{Hétéronomie, détour de production et contre-productivité}

Elaboré dans les années soixante-dix, le cadre conceptuel défini par Ivan Illich dans sa critique radicale de la société industrielle distingue deux modes de production des biens et services. En effet, je peux me déplacer à pied mais aussi en avion ; produire mon alimentation ou consommer des plats cuisinés, voire rejoindre une restauration collective; préserver ma santé en adoptant une saine hygiène de vie ou recourir fréquemment au médecin et aux médicaments... Bref, à des modes de production originels qualifiés d'autonomes, se sont substitués progressivement des modes de production dits hétéronomes. Comme nous le verrons dans la suite, cette distinction autonome/hétéronome nous semble particulièrement pertinente en agriculture.

\section{De détour en détour}

L'hétéronomie croissante de production procède de la logique dite du détour [5] : " reculer pour mieux sauter "; autrement dit, cette capacité humaine à opérer des détours pour mieux atteindre les objectifs et les fins que nous nous assignons. C'est ainsi que nous épargnons pour investir dans un matériel plus performant; nous sélectionnons de nouvelles variétés végétales pour améliorer l'efficacité technique... II est clair que la recherche de productivité est la fin affichée du détour de production qui, de fait, va revêtir une double dimension, technique et économique. II s'agit tout d'abord d'un détour de production technique, suscité par le progrès des techniques. La technique appelant la technique dans son développement, les détours de production vont progressivement s'allonger en incluant d'autres détours de production, en s'emboîtant mutuellement, en se bouclant... ce qui contribuera à déployer les modes hétéronomes de production, ceux-ci s'effectuant en outre par la médiation du temps et d'autrui.

C'est par cette double médiation, du temps et de l'échange, que le détour de production prend une dimension, ou plutôt une justification, économique. L'hétéronomie de production opère en effet le passage d'une économie proprement domestique, voire autarcique, à une interdépendance économique de plus en plus large, complexe et diffuse. La mondialisation des échanges agroalimentaires en est un bon exemple.

\section{Saisir le moment contre-productif}

Ce sont en définitive les impératifs économiques qui régissent le passage d'un mode autonome à des modes hétéronomes de production. Afin de préciser ce point, reprenons la manière dont Ladrière ([6], p. 214) distingue l'ordre technique de l'ordre économique par le biais des catégories moyens/fins : la technique s'affaire avec une pluralité de moyens en vue d'une fin déterminée ; alors que l'économie vise à gérer des moyens et ressources limités en vue de fins plurielles. Entre technique et économie, il apparaît donc en quelque sorte une inversion du rapport entre les fins et les moyens. La logique du détour de production contribue en sus à un émiettement de ces fins, en fins intermédiaires notamment, c'est-à-dire en des moyens. Parallèlement, la technique se voit subordonnée entièrement à l'économique; tandis que l'économique se mue en technique, délaissant par là même la qualification morale des fins qu'elle est censée honorer.

Du constat précédent, nous pouvons saisir le premier moment de ce qu'llich dénomme la contreproductivité : à savoir celui de la confusion, puis de l'inversion des fins et des moyens. C'est-à-dire encore de ce moment singulier où la technique et l'économique procèderaient alors d'un même 
mouvement, d'un même moteur. La contre-productivité émerge dans la mesure où les productions et consommations intermédiaires, induites par l'hétéronomie de production, génèrent à leur tour des externalités, c'est-à-dire des interactions entre producteurs et/ou consommateurs qui échappent, originellement du moins, à l'intentionnalité, à l'échange marchand, à une marchandisation explicite. C'est évidemment le cas des pollutions agricoles et industrielles, de la détérioration des conditions sociales liées à la division du travail... En d'autres termes, il s'agit d'externalités négatives qui se transcrivent progressivement en coûts externes, en coûts sociaux caractéristiques de la contre-productivité. Alors qu'à l'inverse, les modes autonomes de production sont plutôt dispensateurs d'externalités positives (le déplacement à pied ou à vélo est plutôt favorable à la santé, l'association de l'agriculture et de l'élevage coproduit un paysage généralement apprécié...), c'est-à-dire de bénéfices externes qui seront, eux aussi, imparfaitement valorisés par la dynamique marchande, ce qui pérennisera « en creux » I'hétéronomie de production.

Cette contre-productivité se manifeste plus généralement par un ensemble d'observations synthétisées par Dupuy [7] de la manière suivante: "Passés certains seuils critiques de développement, plus croissent les grandes institutions de nos sociétés industrielles, plus elles deviennent un obstacle à la réalisation des objectifs mêmes qu'elles sont censées servir : la médecine corrompt la santé, l'école bêtifie, le transport immobilise, les communications rendent sourd et muet, les flux d'information détruisent le sens, le recours à l'énergie fossile, qui réactualise le dynamisme de la vie passée, menace de détruire toute vie future et, last but not least, l'alimentation industrielle se transforme en poison. Nous y sommes. »

\section{Contre-productivité agricole}

Sans aller jusqu'à considérer l'alimentation industrielle comme un poison (!), le développement agricole de ces dernières décennies fournit un "bel » exemple, combinant plusieurs effets contreproductifs et cumulatifs. Dans son versant économique tout d'abord, où il apparaît certains bouclages de cycles de production qui confinent à l'absurde ou du moins à un énorme gaspillage. Estce le cas encore des excédents laitiers, transformés en poudre de lait moyennant une forte dépense énergétique et qui, faute toujours d'acquéreurs, reviennent sur l'exploitation agricole pour nourrir... les veaux à l'engraissement - cet exemple était particulièrement patent avant la mise en œuvre des quotas laitiers mais il n'a pas perdu toute son actualité ? Ce cycle de production se pérennise moyennant un système de subventions et de prix administrés tels que chaque opérateur économique situé tout au long de la chaîne y trouve son compte et, parfois, des rentes de situation. Considérons ensuite les techniques agricoles qui, dès l'origine, visèrent à maîtriser des risques climatiques, productifs et sanitaires. La stabilisation puis l'accroissement de la production et de la productivité s'obtinrent par une sélection empirique de variétés végétales et de races animales, l'association de l'agriculture et de l'élevage, les rotations culturales, qui cédèrent la place ensuite au génie génétique, à la fertilisation minérale, à l'irrigation et à l'utilisation des pesticides comme assurance-récolte. C'est ainsi que les réponses techniques à des risques productifs ont généré de nouveaux types de risques environnementaux avérés : pollutions diffuses, raréfaction de ressources semi-renouvelables telles que l'eau et les sols, auxquels tentent de répondre de nouvelles techniques, telles que l'utilisation d'espèces végétales génétiquement modifiées, qui produiraient selon certains une surenchère non seulement de risques mais d'incertitudes environnementales. Ces 
nouveaux risques, ainsi que le changement d'échelle - du local au global - qui les accompagne, expriment le caractère contre-productif de techniques agricoles censées originellement les maîtriser.

Ce n'est souvent qu'à l'occasion d'un nième détour de production que s'effectue la prise de conscience, individuelle et collective, de la contre-productivité. Cette prise de conscience est d'abord niée, puis repoussée ou esquivée. N'oublions pas en effet que chaque détour de production induit des emplois, des rentes de situation, des reconnaissances sociales, et donc des rigidités contrecarrant des réformes à terme incontournables. L'hétéronomie crée en outre des dépendances économiques : les détours de production étant mis bout à bout comme dans un jeu de dominos, il suffit d'en ébranler un seul pour déclencher une onde de choc sur l'ensemble. Les relations techniques et économiques entre cultures et élevage illustrent une hétéronomie particulière que nous nous proposons maintenant d'étudier de plus près.

\section{Entre cultures et élévage : l'échelle au détriment de la gamme}

Rapportée à la sphère agricole, la dualité entre autonomie et hétéronomie des modes de production se ramène pour l'essentiel à celle préexistant entre, ce que nous dénommerons dans la suite, la gamme et l'échelle de production. L'hypothèse posée ici est qu'en privilégiant et en pérennisant parfois indûment l'échelle au détriment de la gamme, l'économie et la politique agricoles peinent aujourd'hui à présenter une posture de durabilité des systèmes de production.

\section{Bref historique}

Un regard historique sur l'évolution des systèmes de production agricole nous enseigne que la coordination d'activités interne à l'entreprise agricole préexistait à l'apparition généralisée des atteintes portées à l'environnement. Utilisé aujourd'hui comme référentiel, le modèle polycultureélevage se situe comme l'archétype schématique de cette coordination qui s'exprime pour l'essentiel dans le mode de gestion du facteur foncier. Dans ce modèle, les productions animales sont associées aux cultures : la disponibilité de sous-produits végétaux comme aliments du bétail, l'utilisation des déjections animales pour le maintien de la fertilité organique des sols, traduisent cette coordination d'activités interne à la firme agricole. Les coproduits d'une ligne de production sont ainsi utilisés comme intrants pour une autre activité productive. Ces synergies techniques se traduisent par des économies de coûts et s'expriment encore sous d'autres modalités: les rotations culturales (la succession temporelle des cultures sur une même parcelle de terre) contribuent à briser efficacement les cycles de reproduction de parasites animaux et végétaux, ce qui constitue ainsi un facteur de protection phytosanitaire, complémentaire de l'apport des pesticides. En outre, I'utilisation de ces diverses synergies techniques par l'entreprise agricole est dans certains cas créatrice d'externalités positives dont est bénéficiaire la collectivité en général. Tel est le cas de la production et de l'entretien de paysages; le caractère esthétique de certains bocages est lié à des systèmes de production agricole alliant par exemple l'élevage à une utilisation spécifique de l'espace. Conjointement à cette fonction esthétique, d'autres externalités positives sont issues de l'utilisation d'économies internes à la firme, recouvrant plutôt des fonctions écologiques et de maîtrise des risques naturels: biodiversité, niches écologiques, conservation et protection des sols contre l'érosion, régulation de la dynamique des eaux, etc. 


\section{Progrès technique et hiérarchie des prix administrés}

Dans l'immédiat après-guerre, l'agriculteur trouvait encore un intérêt économique à l'utilisation des synergies techniques précédentes, intérêt qui aurait pu être révélé notamment par la présence d'économies de gamme. Ces économies apparaissent dès lors que le coût de production jointe de plusieurs biens est inférieur au coût de production de ces mêmes biens produits séparément : c'est l'association des productions (la gamme de produits) qui induit une économie de coût trouvant des origines très diverses : complémentarités de production liées à l'existence de produits joints (grain et paille des céréales), facteurs quasi-publics (c'est-à-dire certains facteurs qui, s'ils sont acquis pour la production d'un bien, sont disponibles à moindre coût pour la production d'autres biens. Par exemple, certains matériels agricoles)...

Les innovations technologiques et la hiérarchie des prix agricoles administrés qui ont présidé ensuite à la construction de la PAC, ont contribué à l'abandon des diverses complémentarités de production évoquées précédemment. Ainsi, le soutien des prix céréaliers favorisa l'adoption d'innovations technologiques intervenant comme substituts des complémentarités existantes : tel est le cas de I'utilisation accrue des pesticides, assurant ainsi une protection des cultures plus efficace que les simples rotations culturales. Dans un autre domaine, le coûteux processus d'amélioration génétique conduit à des espèces ou des variétés qui, pour exprimer leurs potentialités, nécessitent des intrants très spécifiques. Ceci écarte parfois certains coproduits autrefois réintroduits comme inputs : sousproduits végétaux, déjections animales utilisés comme fertilisants, etc. En outre, la désutilité, voire la pénibilité du travail, associée parfois à l'utilisation des complémentarités de production n'a fait qu'accélérer l'adoption des innovations technologiques.

De manière générale, la croissance de la production agricole depuis la fin des années soixante s'est accompagnée d'un double mouvement d'intensification et de spécialisation des productions, tant au niveau de la ferme qu'au sein de divers bassins régionaux de production. Le processus d'intensification correspond au déploiement des économies d'échelle, c'est-à-dire une diminution du coût unitaire de production, à mesure notamment de la croissance des surfaces d'un côté, des effectifs animaux de l'autre. Quant à la spécialisation des productions, elle peut s'expliquer partiellement par une érosion progressive des économies de gamme, du moins au niveau de l'entreprise agricole.

Parallèlement, le système des prix garantis et la forte innovation technologique induite de ces dernières décennies ont soustrait à l'entreprise agricole une large part de l'emboîtement comme de la coordination d'activités de production qu'elle assurait traditionnellement. Cette part se trouve désormais assurée par des firmes industrielles situées en amont et en aval de l'agriculture qui ont, de fait, ôté une part de la multifonctionnalité dévolue traditionnellement à l'agriculture. La contribution de l'entreprise agricole au process agricole et agro-alimentaire se trouve ainsi amoindrie, de même que les aménités environnementales évoquées précédemment. L'annulation de ces aménités s'interprète parfois comme des externalités négatives, selon bien sûr les divers modes d'attribution des droits de propriété qui régissent ces externalités. A celles-ci s'ajoutent : le caractère désormais indésirable de certains coproduits (déjections animales); l'utilisation massive des pesticides et des engrais chimiques qui provoque des atteintes à la biodiversité; leur accumulation dans les écosystèmes qui peut occasionner à terme des effets nocifs sur la santé humaine; le maintien problématique de la fertilité des sols, voire de leur conservation. Bref, tous ces méfaits 
environnementaux liés à l'intensification et à la spécialisation laissent à penser que si les économies de gamme ne subsistent plus en termes de coût privé de production (i.e. le prix de revient pour le producteur), elles subsistent par contre en termes de coût social, résultante du coût privé de production et de l'ensemble des externalités négatives et positives associées.

\section{Politique agricole : entre découplage et recouplage}

Si l'association agriculture-élevage constitue l'archétype de productions naturellement jointes, leur dissociation opérée par l'hétéronomie croissante des modes de production a vraisemblablement facilité le découplage des aides publiques à l'agriculture, tel celui entériné en juin 2003 par l'accord de Luxembourg sur une nouvelle réforme de la Politique Agricole Commune (PAC). De ce fait, cette nième réforme de la PAC a mimé la logique libérale du Fair Act américain de 1996 qui voulait rompre avec une politique interventionniste œuvrant depuis les années trente aux Etats-unis. Dès 1998 cependant, l'Etat fédéral a dû intervenir massivement par des aides d'urgence, suite notamment à des calamités naturelles et de fortes baisses des prix. Prenant acte de cette déconvenue, le Farm Security and Rural Investment Act (FSRIA) de 2002, succédant au Fair Act de 1996, a supprimé la dégressivité des aides découplées. II institutionnalisait par ailleurs des aides d'urgence, sous forme de paiements dits contracycliques attribués sur des surfaces de référence et déclenchés automatiquement lorsque le prix moyen à la production est inférieur à un prix d'objectif défini par le FSRIA... Soit donc un recouplage partiel de l'aide à la surface cultivée qui devrait aboutir dans les dix ans à une augmentation de près de $80 \%$ du budget de l'agriculture américaine.

\section{Travers économiques, travers éthiques}

Malgré l'âpreté de ce constat, il faudra encore quelque temps pour reconnaître que le découplage des aides publiques à l'acte de production n'est pas une panacée. Pour l'heure, il reste encore le maître mot des économistes agricoles, se définissant comme la caractéristique d'une politique publique (de soutien des revenus, agri-environnementale...) qui n'affecterait en rien les niveaux de production et d'échanges marchands. Deux commentaires, l'un plutôt économique et l'autre plutôt éthique, peuvent être associés à cette définition.

D'un point de vue économique, il vaudrait mieux donc coupler l'aide publique aux efforts de préservation environnementale plutôt qu'aux volumes produits. C'est ce qu'on entend encore par écoconditionnalité : cela ressort de l'évidence, du bon sens et ne peut que restaurer l'équité... En attendant ce jour, le couplage à des objectifs environnementaux s'avère difficile dans la pratique, peu efficace et coûteux d'un point de vue budgétaire, à moins qu'il ne permette de pérenniser un encadrement agricole et para-agricole dont les effectifs en termes d'emploi n'ont pas suivi pour l'instant la décrue du nombre d'exploitations agricoles. Notons également que les aides découplées se transcrivent rapidement en droits à produire qui sont ensuite marchandisés par le biais des transactions foncières, ce qui alourdit financièrement l'installation des jeunes. Le découplage dissocie enfin davantage les fonctions agricoles productive et environnementale qui demeurent, qu'on le veuille ou non, des activités jointes ${ }^{2}$.

En arrière-plan de la définition précédente du découplage, transparaît cette subordination du politique à l'économique, une politique réduite à faciliter l'expression de la "vérité " et de la transcendance de l'échange marchand désormais mondialisé : tel est le "bien » à faire dans ce nouvel ordre tout à la fois mondial et moral. Pour s'en convaincre, il suffit d'observer le langage 
moralisateur des économistes : n'ont-ils pas pour mission première de dénoncer des politiques qui "affectent" la libéralisation des échanges? Ces mêmes politiques qui introduisent des " distorsions ", qui se refusent à la " discipline " prônée par le marché lui-même, s'exposant ainsi aux " sanctions " de ce dernier, au grand dam du plus grand nombre. Plus qu'un ordre moral, il s'agit d'une transcendance objet de croyance et de crainte. Comme tout faux dieu qui se respecte, une telle transcendance convie au fatalisme - à quoi bon se dresser face à l'anonymité des échanges marchands ? - ou à de nouvelles jacqueries menées par les mouvements alter-mondialistes.

Divinisation ou diabolisation, la vérité, nous semble-t-il, est ailleurs. A commencer par le fait que la dynamique marchande ne fait qu'agréger et amplifier les propres travers des politiques publiques. En délivrant parfois des prix mondiaux inférieurs aux coûts de production, les marchés agricoles ont pris en compte le fait que les pays développés ne sont pas prêts demain à lâcher leur agriculture. D'où la déception, voire l'agacement, de pays agro-exportateurs tels que le Brésil ou encore des pays africains pour l'exportation de coton, qui ont le sentiment récurrent d'être conviés à un marché de dupes. Ceux-ci en effet n'ont pas seulement les moyens de soutenir leur agriculture mais espèrent plutôt en retirer des ressources monétaires pour leur propre développement. Comme l'a montré d'ailleurs l'échec de la conférence ministérielle de Cancun en septembre 2003, la confrontation commerciale se positionne désormais vis-à-vis de ces pays, et de ceux qui auront demain un poids majeur dans la configuration mondiale des échanges agricoles : la Chine, l'Inde...

Pour autant, la situation n'est pas " tout blanc, tout noir »! Face au dumping à l'exportation des USA comme de l'Union européenne, les nouveaux pays agro-exportateurs n'ont parfois d'autre recours que celui d'un dumping écologique qui se traduit par une surexploitation des ressources en eau, en terres, en forêts. Voire à un dumping social via une très faible rémunération du travail agricole. Certes, un renforcement des législations du travail et de l'environnement peut contraindre leurs exportations à court terme. A moyen terme cependant, l'adoption d'innovations biotechnologiques, souvent mieux socialement acceptées dans ces pays, contribuera à une substitution accélérée du capital au travail... qui promet de belles réserves de productivité au grand dam des pays traditionnellement exportateurs.

\section{Des jointures au recouplage}

Sursaut salutaire ? Dans le cadre de la nouvelle réforme de la PAC adoptée en juin 2003, les Etats membres pourront maintenir un lien avec la production, tel celui souhaité par la France de laisser coupler $25 \%$ des aides aux surfaces en céréales et oléoprotéagineux. Le reste serait découplé ou plutôt "couplé » au respect de normes environnementales, sanitaires, de bien-être animal, etc. c'est-à-dire à autant de co-produits joints aux productions animales et végétales mais qui ne sont pas directement valorisables par le marché. Le problème est que la valorisation, pour l'agriculteur, de ces jointures de production suppose en vis-à-vis des « jointures de consommation ». Celles, par exemple, observées pour la consommation de produits issus de l'agriculture biologique, par laquelle sont visées conjointement une consommation proprement alimentaire, un souci de préservation de la santé, voire un souci éthique de préservation de l'environnement ou de soutien d'un mode d'agriculture familiale. Il est clair que la correspondance entre jointures de production et jointures de consommation est un enjeu émergent des politiques publiques de l'agriculture qui nécessiteront des travaux de recherche complémentaires. Ceux-ci restent encore trop focalisés sur l'économie de la production, omettant ainsi que les jointures de production sont en réalité soutenues par les 
structures des fonctions d'utilité. En définitive, cette fameuse multifonctionnalité de l'agriculture est "soutenue" par la structure de la fonction d'utilité des consommateurs, qui n'est autre que l'expression duale de la jointure dans le langage de la micro-économie.

Plus prosaïquement, on pressent bien qu'une correspondance efficiente entre jointures de production et jointures de consommation nécessitera des marchés de proximité (je souhaite profiter des biens publics coproduits par l'agriculture), voire dans certains cas un couplage partiel du soutien public à l'acte de production. A l'inverse, l'internationalisation des marchés conduit à une anonymité des échanges et permet en conséquence de s'affranchir sournoisement de la caution éthique liée aux actes de production comme de consommation. Le maintien de la correspondance entre jointures de production et de consommation nécessitera tout à la fois une maturité des comportements d'achat et une organisation plus fine du marché lui-même, telles celles que l'on observe dans les pratiques de commerce équitable. C'est à ce prix seulement que le découplage pourra se déployer efficacement, laissant la place à une juste rémunération du travail agricole, quelle que soit la latitude sous laquelle il est dispensé.

\section{Réarticuler autonomie et hétéronomie de production : quelques perspectives}

Injonction aujourd'hui incontournable des politiques agricoles, le découplage des aides publiques entérine en définitive l'incapacité des marchés agricoles à rendre un juste prix, résultant d'une juste combinaison entre efficacité et équité. A ce propos, il serait opportun d'approfondir la signification éthique du découplage des aides agricoles, à en dégager une véritable herméneutique. En effet, le découplage économique ne se double-t-il pas d'un découplage " éthique " qui affranchit producteurs et consommateurs de la nécessité d'apposer une dimension morale à leurs décisions économiques ? Cette démission éthique se traduit par un coût social : en un mot comme en mille, tout se passe comme si les économies d'échelle procurées par l'accaparement parfois violent des marchés se dissipaient dans la démultiplication des rentes foncières et des coûts de transaction liés aux mesures agri-environnementales.

Entre échelle et gamme de production, le choix n'est donc pas seulement économique mais se double d'une dimension morale, concernant tant les producteurs que les consommateurs. C'est ce que nous avons voulons signifier ici, notamment dans cette correspondance à rechercher et à favoriser entre jointures de production et jointures de consommation. Privilégier à nouveau la gamme de production revient en fait à réarticuler autonomie et hétéronomie de production en vue d'une meilleure efficience sociale et environnementale. A cette fin, il conviendra d'encourager l'évaluation des économies de gamme, une évaluation qui demeure néanmoins délicate. En comparant deux coûts de production, la mesure des économies de gamme se place d'emblée entre le niveau micro-économique de l'entreprise (l'exploitation agricole) et celui quasiment d'une ou de plusieurs branches. Alors que l'entrepreneur agricole comprend les économies de gamme en termes de coût privé, le décideur public se doit de les mesurer en termes de coût social, par l'intégration des externalités positives et négatives. Ainsi, une situation de deséconomies de gamme mesurées en termes de coût privé peut se convertir en économies de gamme positives si celles-ci sont mesurées en termes de coût social. Restreindre la mesure des économies de gamme en termes de coûts privés de production peut cautionner en effet à terme le dumping écologique. 


\section{Références}

1 OCDE. L'agriculture dans un monde en mutation : quelles politiques pour demain ?. Paris : Réunion du Comité de l'agriculture au niveau des ministres, Communiqué de presse, 5-6 mars 1998.

2 VERMERSCH D. L'agriculture entre artificialisation des milieux et artificialisation des échanges. OCL $2000 ; 7(6): 480-4$.

3 VERMERSCH D. La multifonctionnalité : une synthèse des travaux français récents. 2001 ; Rapport pour l'OCDE, INRA-ESR-Rennes 2001/04.

4 VERMERSCH D. Le découplage des aides agricoles : so what ? Les Echos. 4 décembre 2003.

5 DUPUY J-P. Le détour et le sacrifice Ivan Illich et René Girard. Esprit 2001 : 26-46 ; Mai.

6 LADRIÈRE J. L'éthique dans l'univers de la rationalité. 1997 ; Artel-Fides.

7 DUPUY J-P. Ivan Illich ou la bonne nouvelle. Le Monde 2002 ; 27 décembre.

8 HEADY EO. Economics of Agricultural Production and Resource Use. NJ : Prenctice-Hall : Englewood Cliffs, 1952.

9 BEATTIE B, TAYLOR CR. The Economics of Production. New York : John Wiley \& Sons, 1985.

10 VATN A. Multifunctional agriculture : some consequences for international trade regimes. European Review of Agricultural Economics 2002 ; 29(3) : 309-27.

11 LEATHERS H. Allocable Fixed Inputs as a Cause of Joint Production : A Cost Function Approach. American Journal of Agricultural Economics $1991 ; 73: 1083-90$.

12 PANZAR J-C, WILLIG R-D. Economies of Scale and Economies of Scope in Multi-Output Production. Bell Laboratories Economic Discussion Paper n³3, 1975.

13 PANZAR J-C, WILLIG R-D. Economies of Scope. American Economic Review $1981 ; 71(2)$ : 268-72.

1 Externalité : se dit d'une situation où une interaction entre agents échappe originellement au marché ; un bien public quant à lui est un bien qui autorise une consommation collective. $2 \mathrm{~A}$ ce propos, on s'intéressera aux travaux récents de l'OCDE s'évertuant à minimiser les possibles jointures de production, justifiant ainsi des politiques agricoles découplées. 